\title{
A Causal Model to Analyze Aircraft Collision Avoidance Deadlock Scenarios
}

\author{
Miquel Ángel Piera Eroles ${ }^{(a)}$ \\ (a) miquelangel.piera@uab.cat \\ Julia de Homdedeu $^{(b)}$, Maria del Mar Tous ${ }^{(\mathrm{c})}$, Thimjo Koca ${ }^{(\mathrm{d})}$, Marko Radanovic ${ }^{(\mathrm{e})}$ \\ (a),(b),(c),(d),(e) Department of Telecommunications and Systems Engineering \\ Autonomous University of Barcelona \\ Barcelona, Spain \\ (b) juliade.homdedeu@e-campus.uab.cat, ${ }^{(c)}$ mariadelmar.tous@e-campus.uab.cat, \\ ${ }^{(\mathrm{d})}$ thimjo.koca@uab.cat, ${ }^{(\mathrm{e})}$ marko.radanovic@uab.cat
}

\begin{abstract}
Continuous increase in the traffic density over the certain en-route sectors provokes many situations in which a loss of separation minima (SM) between two aircraft occurs. Although, this loss is predicted well in advance, giving a proper look-ahead time (LAT) for a detection function, the resolution of such an event may lead to a new conflict situation due to dynamics of surrounding traffic aircraft. A multiagent system framework can deal with these cases. This work presents three different complexity indicators that can be used to shape the social behavior of the agents. Simulation results show that the proposed indicators can suggest drastically different nature of the same ecosystem, therefore further investigation of the correlation of the proposed indicators to the actual complexity is necessary.
\end{abstract}

Keywords: ecosystem, feasible solutions, opportunity costs, conflict maneuvers

\section{INTRODUCTION}

Continuous increase in the traffic density over the certain en-route sectors provokes many situations in which a loss of separation minima (SM) between two aircraft occurs [2,4]. Although, this loss is predicted well in advance, giving a proper look-ahead time (LAT) for a detection function, the resolution of such an event may lead to a new conflict situation due to dynamics of surrounding traffic aircraft $[5,8]$. Namely, some resulting maneuvers of the conflicting aircraft can induce new loss of SM with nearby aircraft in which new LAT for detection can be significantly reduced. Consequently, a collision risk in this case is often at a higher level, which usually requires more demanding avoidance maneuver for the pilot-in-command, generating also inefficient trajectory segments.

At present, an upgraded Traffic Alert and Collision Avoidance System (TCAS II v7.1), has been designed for operations in the traffic densities of 0.3 aircraft per squared nautical mile $[1,8]$. It demonstrates excellent performances for the pairwise encounters, as well as the great improvements for multi-thread encounters, taking different flight configurations (cruising and evolving aircraft) into considerations. However, a TCAS logic shows some operational drawbacks due to limited number of resolution advisories, currently resulting in the vertical flight profile change only [3,7]. Moreover, the well reported induced collisions in many traffic scenarios show a high probability of occurrence. Thus, there is a challenge to investigate and implement a new operational framework which will improve and extend TCAS functionalities at both tactical and operational level.

This paper relies on a new research in the ATM automation framework: the concept of ecosystems $[1,6]$. Ecosystem presents a set of aircraft, with selfautomated capabilities, that form a cost-efficient separation management system for finding the best compromise in resolution trajectories. The goal is to transform the ecosystem aircraft into intelligent agents that can communicate with each other to safely make the best use of existing airspace capacity. The concept has been developed to handle a robust conflict management process considering aircraft performances, and consistent solutions in front of the scalability problems.

The study introduces the importance to analyze the interdependencies between aircraft to predict the available time for a negotiation process between the air space users involved in the surrounding traffic of 
a conflict before a compulsory resolution is issued by the air traffic controller (ATC).

\section{BACKGROUND}

The current ATM is basically a ground-based system with different levels of operational management tasks: Airspace Management; Air Traffic Flow and Capacity Management (ATFCM), which is also in charge for the Trajectory Management (TM); and finally, Separation Management (SM), developed by the ATC, in order to provide safe and smooth operations for all airspace users.

Additionally, for those situations when the ATC has been unable to remove conflicts and a separation management infringement occurs, a different safety net exists for collision avoidance (CA). This safety net is independent from ATC and is an "on board", pairwise based and coordinated resource. Currently is conducted by the TCAS/ACAS.

Nowadays, the transition from the SM function to TCAS is done in a disruptive manner by means of the issuance of a TCAS Resolution Advisory (RA). As a result, crew shall suddenly stop following controller instructions and follow those provided by the onboard system. That involves important operational discontinuities and a very poor level of integration between both safety management layers, SM and CA. Furthermore, when facing a CA situation, the TCAS safety net recognises that for aircraft $(\mathrm{A} / \mathrm{C})$ densities above $0.3 \mathrm{~A} / \mathrm{C} / \mathrm{NM}^{2}$, a sequential pairwise resolution can induce collateral conflicts in surrounding areas. Therefore, conventional and pairwise CR for clusters (CL) of aircraft is highly limited and dependent of the traffic density and complexity.

Besides, for the future ATM system, the Performance Based Operation (PBO) concept proposes a higher degree of integration between $\mathrm{A} / \mathrm{C}$ operation management levels and actors, promoting more dynamic task allocations and continuity between them. The TM is understood an activity performed before $\mathrm{A} / \mathrm{Cs}$ are flying, devoted to deliver agreed flight plans among all the stakeholders, whereas SM is assumed as those reactive measures to be implemented anytime and anywhere the separation minima between an $\mathrm{A} / \mathrm{C}$ and a hazard is under risk. The TM and the SM functions continuously conduct a trade-off between efficiency and safety in order to avoid jeopardising the performance of the system but maintaining high safety levels.

AGENT project is devoted to facilitate the integration between SM and CA activities, questioning the current fixed task allocation on the ground for separation management, and on the air for autonomous collision avoidance. To this end, a proactive SM management is proposed supported by multi-agent task allocation, where the "separator's" function will be performed by the ATC, but $\mathrm{A} / \mathrm{Cs}$ involved in the safety issue will have an active role in the decision making process through a negotiation process supported by agent technologies. In addition, AGENT also seeks for an enhanced integration among the TM and SM layers by means of a more efficient delivery of trajectory changes (TM) or manoeuvres (SM), for simultaneously planning the resolution of all detected conflicts and potential induced collisions for all aircraft in an efficient and agreed manner.

Agent technology provides the baseline for the implementation of a flight efficient, safe collaborative and supervised separation management, operationally integrated with trajectory management and collision avoidance within a PBO concept. In order to enhance the use of a MAS (Multi Agent Simulation) model to validate a more pro-active role of airspace users in conflict resolution tasks through a negotiation process, it is required a proper time-stamp analysis of perishable speed resolutions.

\section{MAS NEGOTIATION PROCESS}

Aircraft and ATC will be considered intelligent agents within a Multi-Agent System for decision making, in which the number of aircraft starts with the elemental case of a pair-wise conflict, but without being bounded as the number of aircraft will depend on the traffic configuration and the considered complexity for establishing the ecosystem membership.

The Ecosystem tracking (ET) and resolution is performed collaboratively by the ATC and involved $\mathrm{A} / \mathrm{Cs}$, although ATC is always considered the main separator. ATC ground tools conduct the synchronization and updating of trajectories, the evaluation of the ecosystem state and the generation of potential resolution trajectories by exhaustive analysis of the state space for all ecosystem members as well. On the other hand, $\mathrm{A} / \mathrm{Cs}$ will negotiate to find a commonly agreed conflict-free solution for all members while the ATC will monitor the negotiation evolution to be ready for a potential deadlock. In case the negotiation reaches the deadlock state or the evolution of the members is compromising the safety of the operations, ATC will impose a safe solution for all A/Cs. In Figure 1 it is represented the flow diagram only for the negotiation process between agents. 


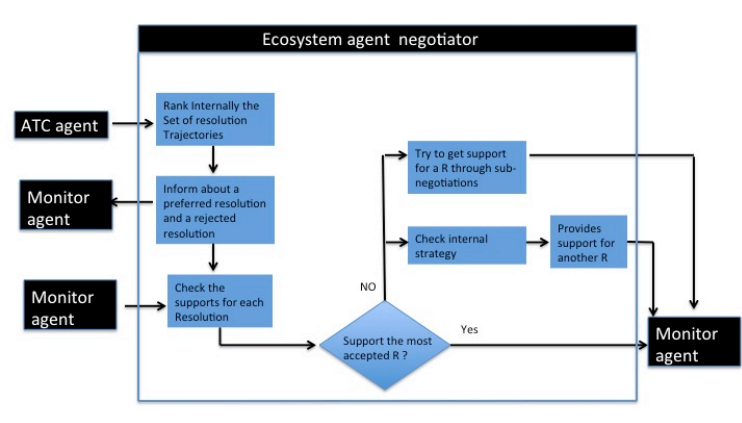

Figure 1: Flow Diagram for the agent negotiation process

In that diagram, the decision about when a consensus is reached or the ATC must issue a compulsory resolution trajectories is not represented since it is considered that is not part of the communication mechanism between agents, instead is a boundary condition that will allow the monitor agent (MnA) to stop the negotiation loop. As it can be observed, the business model of the AU and the negotiation strategy of each agent is a hidden information, the 2 key shared date between agents during negotiation are:

1. Preferred Resolution: The negotiator agent represents a particular aircraft that belongs to the ecosystem and knows the business model of the company. Thus, he can easily rank all the conflict resolution combinations generated by the ATC using the state space analysis tool considering AU preferences. As a result of this internal ranking and the information provided about the acceptability of the different resolutions by the other agents, it advert a particular resolution that would receive his support.

2. Rejected Resolution: Among the different resolutions provided by the ATC agent, the negotiator agent has the capacity to block one resolution since according to the business model of the company this resolution could affect negatively its preferences. This information is used by the rest of agents to relax its preferences in order to find a consensus before a compulsory resolution is issued by the ATC.

Among the feasible combinations of resolutions that are provided by the ATC agent, the deadline for the negotiation could be (or not) provided in order to force a consensus in a short time and avoid the ATC intervention. Note, that different negotiation policies could be supported by means of this flow diagram. For instance, if the deadline for the negotiation loop is provided to the agents at the first negotiation loop, some agents could take the advantage to delay a consensus to force a resolution that could provide better benefits. On the other way around, the lack of the deadline for the negotiation, could also help to reach a consensus under "the sooner the better" policy. This former option could work if all the ecosystem member belongs to the same company.

To summarise, the flow diagram and its ontology has been elaborated to support different negotiation policies preserving this information that AU's wouldn't never share, but providing the possibility to reach a consensus among the ecosystem members while at the same time provides enough information to predict if the negotiation is moving towards a consensus or is reaching a negotiation trap that circle around the same arguments.

\section{MAS DEADLOCK}

NEGOTIATION

Among the different factors that can lead to the stagnation of negotiation processes in the resolution of an ecosystem, the most relevant ones that should be considered during the design of the negotiation ontology are:

- Non-cooperation due to different jargon: The agents designed by OD end-users to test different $\mathrm{CR}$ policies should preserve the ontology described in this deliverable.

- Exclusion of some actors in the interaction process: During the Ecosystem Creation function all the cluster members that are willing to be members of the ecosystem and fulfil the equipment requirements to be members of an ecosystem (FUN-CEC-001). To avoid a trap due to a non-cooperative aircraft, the state space will generate only feasible resolutions for those aircraft involved in the negotiation process.

- Conflicting interests and values: At global level, the fierce competition between airlines to block preferred slots and trajectories could lead to a negotiation trap, however, at ecosystem level (i.e. local context), in which a compulsory resolution would be issued by the ATC in which AU's preferences will not be considered, is a positive influence factor to enhance AU's to reach an agreement supporting a balanced trade-off among the different performance interests.

To avoid poor results due to negotiation deadlocks between ecosystem members, an external actor is 
considered in the ontology that represents the ATC with the capacity to interrupt the negotiation process at any time and issue a non-agreed resolution trajectory to some or all the ecosystem members.

Despite the ontology will support the influence of ATC preferences as an external actor during the negotiation process, this capacity does not mean that the external actor is always involved in the resolution process. It is expected that the Open Demonstrator will provide new results about the ecosystem characteristics that usually requires the intervention of the ATC as external actor when the AU's actors who are involved in the deadlock are close to reach a resolution agreement but finally the external actor is necessary to overcome small difficulties

The level of performance that can be achieved in the resolution of ecosystem conflicts is the result of interactions between many entities at different levels. The roles of these various entities must be properly identified considering those agents that just react to the environment with respect to those agents with choice capacity which usually will embed different algorithms to act on the environment trying to reach their goals in an efficient and safe way.

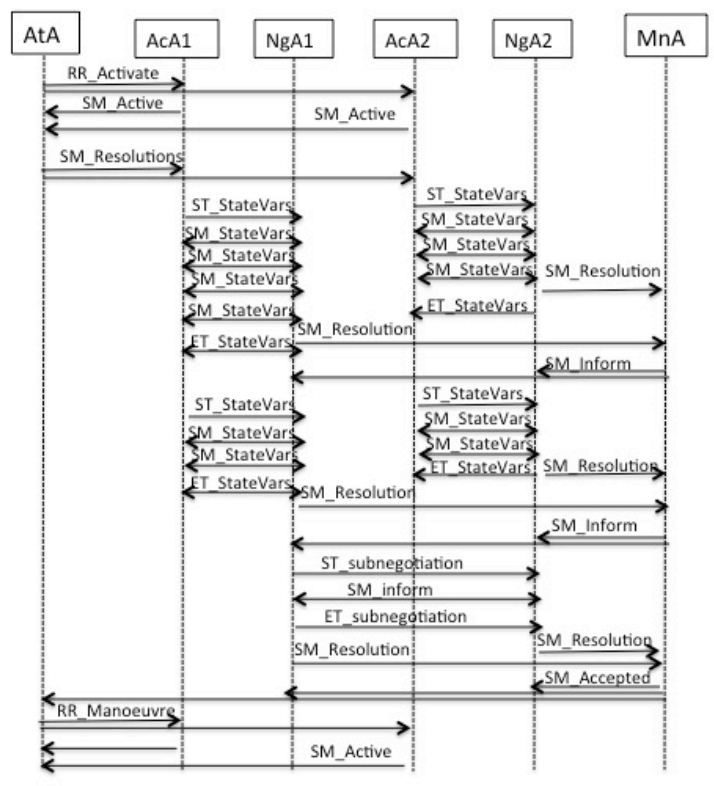

Figure 2: Resolution Consensus after 3 interactions

In Figure 2 it is represented graphically a negotiation process under an Event-oriented policy, in which a resolution consensus is achieved after 3 interactions: the first 2 interactions are supported by negotiator agents providing their preferred and no-go resolution while the third interaction is through a direct subnegotiation process between negotiators agents.
For simplicity only 2 aircraft are considered in the negotiation loop. The steps in the negotiation are:

1. Initially, the ATC Agent sends a RequestResponse message (RR_Activate) to each aircraft of the ecosystem to validate its willingness to participate in the negotiation process to reach a resolution consensus. The ATC agent remains waiting until a response (SM_Active) from each aircraft is received.

2. Once the ATC have the confirmation of the aircraft that belongs to the ecosystem, its computes the combination of feasible resolutions by means of the state space analysis and sends a message (SM_Resolutions) with the resolutions to the aircraft agents and the monitor agent. Note, that the set of feasible resolutions cannot be computed until the cluster aircraft have confirmed they accept to become ecosystem members.

3. Each Aircraft Agent updates its variables states and shares this information through a Start_Transaction message (ST_StateVar) to its negotiator agents who holds the business model to constantly update the state variables of the aircraft together with the costs of some manoeuvres identified by the agent negotiator. The flow of information is finalized by an End Transation message (ET_StateVar) sent by the negotiator agent.

4. Once the negotiator agent has identified the best and the no-go resolution trajectory, it informs the monitor agent by means of a message (SM_Resolution) about the preferred resolution trajectory and the no-go trajectory.

5. Since this synchronization mechanism is an event-oriented mechanism, the monitor agent will wait until all negotiator agents have sent the SM_Resolution message, to crosscheck if there is any resolution consensus among the ecosystem members.

6. In case no consensus is reached, the monitor agent will send a message (SM_Inform) to all the negotiator agents with the most accepted resolutions and the no-go ones. In Figure 3 there are represented only 2 aircraft for simplicity purposes, but in an operational ecosystem the monitor could receive several preferred resolution trajectories and only those that received more support will be used in the SM_Inform message. 
7. In case a consensus is reached, the monitor agent will send a message (SM_Accept) to all the negotiator agents and the ATC agent providing the consensus reached (i.e. resolution trajectories to be implemented by the aircraft).

8. Once the ATC agent has been informed that a resolution consensus has been reached, it validates the manoeuvres and informs each aircraft agent by means of a Request Response message (RR_Manoeuvre) to implement the manoeuvre.

The negotiation loop is finalized once the ATC agent receives from each aircraft agent a message (SM_Confirm) confirming that the resolution will be performed.

\section{RESOLUTION DEADLOCK DYNAMICS AND SIMULATION RESULTS}

In the considered scenarios, some assumptions are made. Firstly, the aircraft trajectories during the existence of the ecosystem are linear segments.

Secondly the maneuver space is discretized in space and time, which means aircraft can perform maneuvers with a certain deviation angle from the original trajectory and these maneuvers can be performed only at discrete time instances. The maximum angle deviation is assumed to be $30^{\circ}$ and the increment $5^{\circ}$. The time increment is taken 1 second (see figure 3).

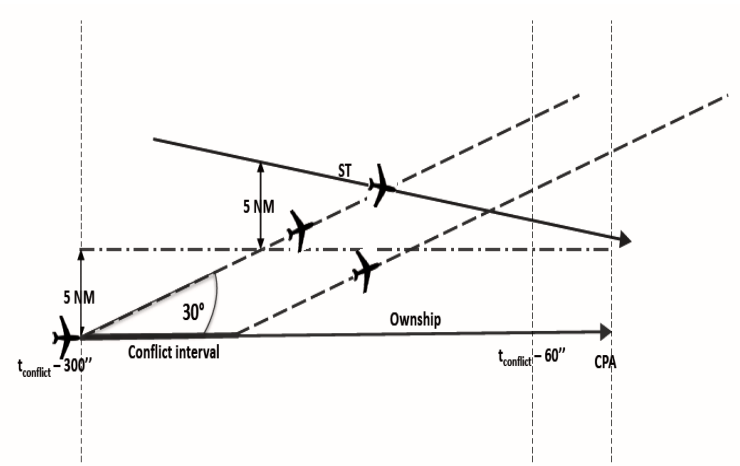

Figure 3: Possible resolutions to be flown by an aircraft

Lastly the possible taken maneuvers should be synchronous, i.e. all the performed maneuvers that will be taken to resolve the conflict should be taken at the same time from all aircraft members.

The ecosystem trajectories are graphically presented in a 3D Euclidean space, with latitude and longitude measured in $[\mathrm{km}]$ and altitude in $[\mathrm{ft}]$. The results show the cumulative number of the feasible trajectories over the ecosystem time
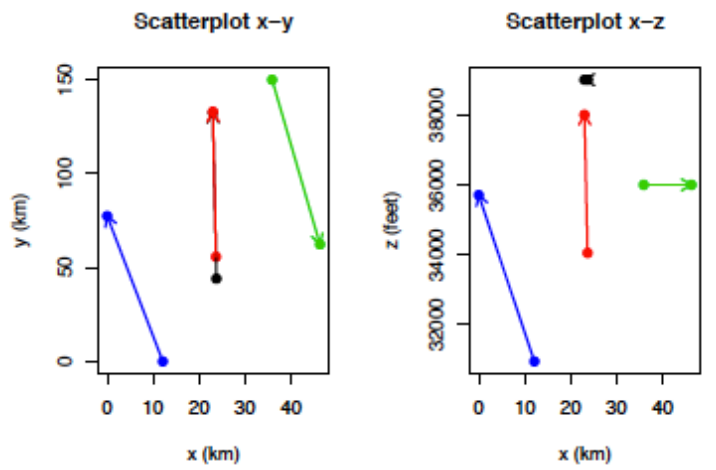

Figure 4: Ecosystem scenario-1

As can be seen from Figure 4, ecosystem 1 is composed of three aircraft in an evolving encounter. It is easy to note in figure 5, that early seconds during the negotiation process is critical to reach a good resolution consensus between ecosystem aircraft. During the first 40 seconds of the negotiation process, the amount of feasible resolutions drops to half of the original set of negotiable resolutions. This characteristic do not depend only on the amount of the aircraft that belongs to the ecosystem, but also to the trajectory configurations.

\section{$\mathrm{N}$ solutions vs time}

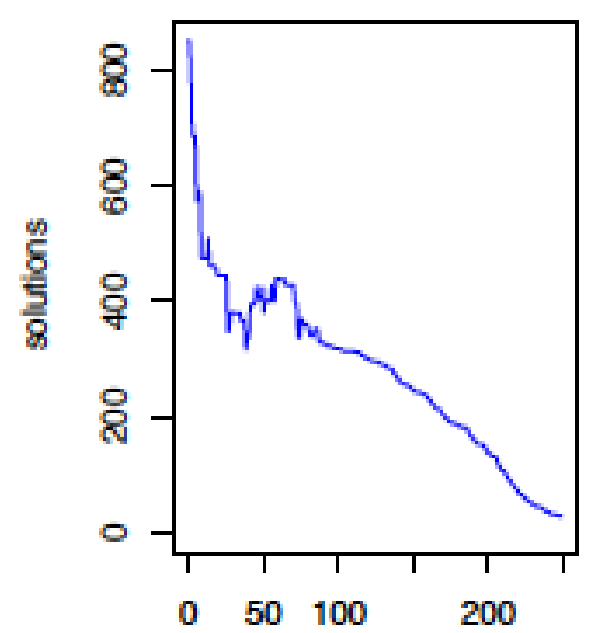

Figure 5: Feasible conflict resolutions in scenario 1

In figure 6, another ecosystem with 5 aircraft is represented. 

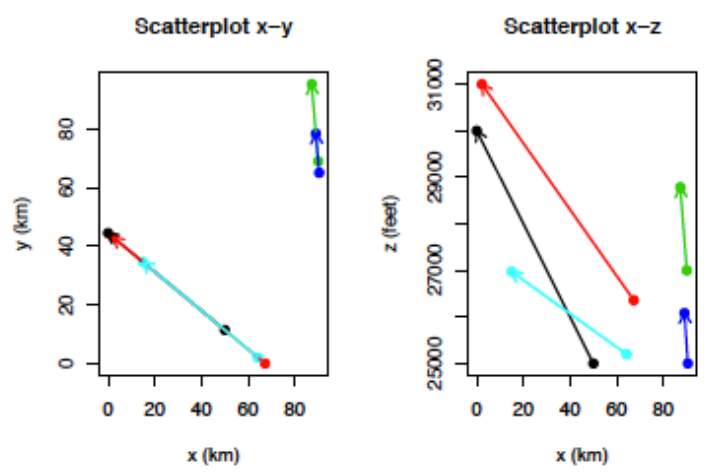

Figure 6 Ecosystem scenario 2

The amount of feasible resolutions is represented in figure 7. As it can be noted, the amount of feasible resolutions increments after 40 seconds since one of the threads in the surrounding traffic leaves the ecosystem.

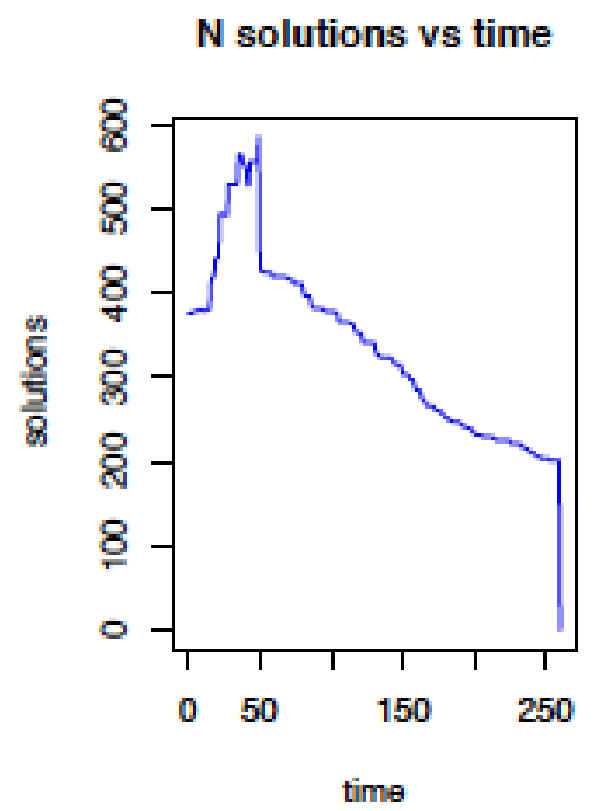

Figure 7: Feasible conflict resolutions in scenario 2

\section{CONCLUSIONS}

This study illustrates the importance of a quantitative analysis of the potential resolution for a given pairwise aircraft conflict considering the surrounding traffic. The speed of perishable resolutions is a critical factor to implement MAS models to support an efficient negotiation process in which the airspace users could take an active role considering hidden business models.

\section{Acknowledgements}

This research is partially supported by the EU Horizon 2020 Research and Innovation Programme, Project " Adaptive self-Governed aerial Ecosystem by Negotiated Traffic (under Grant Agreement No. 699313)" and Ministry of Economy and Competitiveness. Project "Fire Guided Unmanned Aircrafts and Resources Distribution (TIN201456919-C3-1-R). Opinions expressed in this paper reflect the authors' views only..

\section{References}

[1] AGENT Project Team, 2016. Report on AGENT functional and non-functional requirements. Ref. 2015 (699313), Cranfield, United Kingdom.

[2] Bouarfa, S., Blom, H. A., Curran, R. and Everdij, M. H., 2013. Agent-based modeling and simulation of emergent behavior in air transportation. Complex Adaptive Systems Modeling, 1(1), 15.

[3] Enea, G. and Porretta, M., 2012. A comparison of 4D-trajectory operations envisioned for Nextgen and SESAR, some preliminary findings. In: 28th Congress of the International Council of the Aeronautical Sciences, pp. 2328, Sept, 2012.

[4] Kochenderfer M. J., Holland, J. E. and Chryssanthacopoulos J. P., 2012. Next generation airborne collision avoidance system. Lincoln Laboratory Journal, 19 (1), 17-33.

[5] Murugan, S. and Oblah A. A., 2010. TCAS Functioning and Enhancements. International Journal of Computer Applications, 1 (8), 46-50.

[6] Radanovic, M., Piera, M.A., Koca, Th., Saez, F.J., 2017. Airborne Ecosystem Complexity Analysis for Tactical Conflict Management. IEEE Transactions on Intelligent Transportation Systems,

[7] Ramasamy, S., Sabatini, R., Gardi, A. and Kistan, T., 2014. Next generation flight management system for real-time trajectory based operations. Applied Mechanics and Materials, 629, 344-349.

[8] Tang, J., Piera, M. A., Ling, Y. and Fan, L., 2015. Extended traffic alert information to improve TCAS performance by means of causal models. Mathematical Problems in Engineering, 2015.Pedrycz, W., (1993) Fuzzy sets and fuzzy systems, Research Studies Press, England 